\title{
Reducing the Longitudinal Emittance of the 8-GeV Beam via the rf Manipulation in a Booster Cycle
}

\author{
Xi Yang, Valeri A. Lebedev, and Charles M. Ankenbrandt \\ Fermi National Accelerator Laboratory \\ Box 500, Batavia IL 60510
}

\begin{abstract}
Bunch rotation will cause the longitudinal emittance growth whenever there are far more A rf stations than B rf stations, or vice versa. An alternate method via optimizing the RFSUM curve in a Booster cycle has been investigated using the ESME simulation. Since the rf manipulation at transition crossing can reduce the longitudinal emittance $31 \%$ and the momentum spread $17 \%$, eventually, the rms momentum spread of $2.98 \mathrm{MeV}$ and the longitudinal emittance of $0.061 \mathrm{eV} \cdot \mathrm{sec}$ with $95 \%$ of the beam can be achieved at $8-\mathrm{GeV}$.
\end{abstract}

\section{Introduction}

Bunch rotation (BR) via the RFSUM reduction at the end of a Booster cycle has been implemented to reduce the bucket height and area, so the momentum spread $(\Delta p)$ of 18 $\mathrm{MeV}$ and the longitudinal emittance $\left(\varepsilon_{L}\right)$ of $0.1 \mathrm{eV} \cdot \mathrm{sec}$ with $95 \%$ of the beam can be achieved before the beam is transferred from Booster to Main Injector.[1-3] RFSUM is the vector sum of $\mathrm{rf}$ voltages at cavity gaps of all Booster $\mathrm{rf}$ stations. BR requires RFSUM to be reduced from the nominal value of $450 \mathrm{kV}$ to the BR voltage $\left(V_{B R}\right)$ of 150 $\mathrm{kV}$ within 10 to $20 \mu \mathrm{s}$, and afterwards, $V_{B R}$ to be kept as the RFSUM voltage for a quarter of the synchronous period, about $200 \mu \mathrm{s}$, till the extraction. The reasons why it's difficult to keep the phase relationship between the beam and the RFSUM waveform to be constant during BR are: $1^{\text {st }}$, the beam induced voltage (beam loading) intends to shift the 
phase of the RFSUM waveform, and such a shift increases with the decrease of the

RFSUM voltage $\left(V_{R F}\right)$ while the beam induced voltage stays nearly constant; $2^{\text {nd }}$, it takes more than one hundred microseconds for the feedback system of rf stations to compensate the beam loading, and it is slow compared to the BR time; $3^{\text {rd }}$, in the situation that one (or more) rf station is off, the $\mathrm{A}$ and $\mathrm{B}$ balancer needs to be readjusted; $4^{\text {th }}$, and furthermore, the balancer doesn't work very well when there are far more A stations than B stations, or vice versa.[3] The phase slew between the beam and the RFSUM waveform during BR often happens, and it causes $\varepsilon_{L}$ growth of the beam.

It is important for us to develop an alternate method, which can be used to control $\Delta p$ and $\varepsilon_{L}$ of the $8-\mathrm{GeV}$ proton beam. Since $\varepsilon_{L}$ and $\Delta p$ of the beam are determined by the bucket area $(A)$, as shown in eq.1,

$$
A=\frac{16 \cdot \beta}{2 \cdot \pi \cdot f_{r f}} \cdot \sqrt{\frac{e \cdot V_{R F} \cdot E_{s}}{2 \cdot \pi \cdot h \cdot|\eta|}} \cdot \alpha\left(\varphi_{s}\right),
$$

and the bucket height respectively,[4] it is clear that $\varepsilon_{L}$ and $\Delta p$ can be controlled via the $V_{R F}$ curve in a cycle. Here, $\beta$ is the Lorentz's relativistic factor, $f_{r f}$ is the rf frequency, $E_{S}$ is the total energy, $h$ is the harmonic number, $\eta$ is the phase slip factor, $\alpha\left(\varphi_{s}\right)$ is the moving bucket factor,[4] and $\varphi_{s}$ is the synchronous phase.

\section{RF Manipulation}

There are several important factors which need to be considered in the rf manipulation: $1^{\text {st }}$, longitudinal space charge (SC) forces defocus the beam bunch before transition and focus the beam bunch after transition; [4] $2^{\text {nd }}$, since the bunch length (BL) reaches its minimum after transition, and at the same time, SC forces reach its maximum, the circumstance that the strongest repulsive SC forces lead to the particles in the beam approaching each other longitudinally accounts for the negative mass instability; [4] $3^{\text {rd }}$, and above the non-adiabatic transition crossing till BL reaches its minimum, there is a mismatch between the beam bunch and the rf bucket, which excites the BL oscillation (or called quad mode), and afterwards, the bunch starts to rotate inside the bucket and eventually occupies a larger longitudinal phase space.

For the purpose of controlling $\Delta p$ and $\varepsilon_{L}$ of the $8-\mathrm{GeV}$ proton beam, an entire Booster cycle $(33 \mathrm{~ms})$ is divided into three periods: before transition from $0 \mathrm{~ms}$ to $16 \mathrm{~ms}$ 
(BT); transition crossing from $16 \mathrm{~ms}$ to $18 \mathrm{~ms}$ (TR); and after transition from $18 \mathrm{~ms}$ to 33 ms (AT).

During BT, defocusing SC forces try to cancel $\mathrm{rf}$ focusing forces. Whenever SC forces are strong compared to rf focusing forces, there is likely a $\boldsymbol{\varepsilon}_{L}$ growth. In order to avoid (or minimize) the SC-induced $\varepsilon_{L}$ growth, it is important to keep SC forces as low as possible, and this can be done via increasing BL since SC forces are inversely proportional to the cube of BL. Also, since lower $V_{R F}$ is, longer BL is, BL can be maximized via reducing $V_{R F}$ to the value before the beam loss starts.

RF manipulations can be implemented during TR. Since in the longitudinal phase space synchronous motions are frozen during TR, all particles with different momentums have the same revolution period, and any change in the momentum coordinate is almost decoupled from the phase coordinate. So one can increase $\Delta p$ without varying $\mathrm{BL}$ via increasing $V_{R F}$ right before transition. The benefit of increasing $\Delta p$ right before transition is that such a $\Delta p$ increase gives rise to the defocusing force right after transition and keeps the minimum BL away from becoming too short. Usually, when BL becomes too short right after TR, there is a significant $\boldsymbol{\varepsilon}_{L}$ growth due to the negative mass instability.[4] Besides, the circumstance that BL non-adiabatically reaches its minimum after transition brings a mismatch between the beam bunch and the rf bucket. Such a mismatch excites the bunch length oscillation (BLO), and causes $\varepsilon_{L}$ growth. In order to keep BLO minimum, a fast increase in $V_{R F}$ can be applied as a quad kick right after transition, since the increase in $V_{R F}$ at a proper time will generate a defocusing in the momentum coordinate and a focusing in the phase coordinate. Two fast $V_{R F}$ increases, or so called two rf pulses, one right before transition and one right after transition, can be applied to minimize the $\varepsilon_{L}$ growth during the non-adiabatic TR.

During AT, a $V_{R F}$ curve is optimized for the purpose of keeping $\Delta p$ and $\varepsilon_{L}$ of the $8-\mathrm{GeV}$ proton beam minimum. Instead of applying a fast reduction in $V_{R F}$, which is required by $\mathrm{BR}$, to achieve $\Delta p$ of $18 \mathrm{MeV}$ and $\varepsilon_{L}$ of $0.1 \mathrm{eV} \cdot \mathrm{sec}$ (with $95 \%$ of the beam) of the $8-\mathrm{GeV}$ proton beam, a smooth reduction in $V_{R F}$ during the last $10 \mathrm{~ms}$ of a cycle is implemented for the purpose of improving the operational reliability. 


\section{Numerical Investigation}

ESME simulations are used to generate the optimal $V_{R F}$ curve for the purpose of minimizing $\Delta p$ and $\varepsilon_{L}$ of the $8-\mathrm{GeV}$ proton beam.[5] There are several important numerical investigations: $1^{\text {st }}$, the $V_{R F}$ curve during BT needs to provide the maximum injection efficiency and a reasonably long BL in order to keep the SC-induced $\varepsilon_{L}$ growth minimum; $2^{\text {nd }}$, the $V_{R F}$ curve during AT needs to provide the required $\Delta p=18 \mathrm{MeV}$ and $\boldsymbol{\varepsilon}_{L}=0.1 \mathrm{eV} \cdot \sec$ (with $95 \%$ of the beam) for the $8-\mathrm{GeV}$ proton beam; $3^{\text {rd }}$, and two $V_{R F}$ increases during TR need to be optimized in both the time and the amplitude for the purpose of keeping $\Delta p$ and $\boldsymbol{\varepsilon}_{L}$ of the $8-\mathrm{GeV}$ proton beam minimum. All the simulations were done at the beam intensity of $4.1 \times 10^{12}$, including the space charge effect.

First, the $V_{R F}$ curve is optimized for two time periods, BT and AT. $V_{R F} v s$. time in a cycle is shown in Fig. 1(a). Here, the injection time is $0.0 \mathrm{~ms}$. EPSILON vs. time is shown in Fig. 1(b). For a Gaussian distribution, the area containing 95\% of the beam is six time EPSILON. The phase and momentum projections at $8-\mathrm{GeV}$ are shown in Figs. 1(c) and 1(d) respectively. $\Delta p$ (in rms) and $\varepsilon_{L}$ (with $95 \%$ of the beam) of the $8-\mathrm{GeV}$ proton beam are $3.58 \mathrm{MeV}$ and $0.088 \mathrm{eV} \cdot \mathrm{sec}$.

Afterwards, the $V_{R F}$ curve is optimized for the period of TR, while the $V_{R F}$ curve at BT and AT stays the same with the one in Fig. 1(a), at its optimal setting. The $1^{\text {st }} \mathrm{rf}$ pulse is optimized, and the result is: $16.30 \mathrm{~ms}$ to $16.35 \mathrm{~ms}, V_{R F}$ linearly increases from $700 \mathrm{kV}$ to $1000 \mathrm{kV} ; 16.35 \mathrm{~ms}$ to $16.95 \mathrm{~ms}, V_{R F}$ stays at the constant value of $1000 \mathrm{kV}$ (or called flat top); and, $16.95 \mathrm{~ms}$ to $17.0 \mathrm{~ms}, V_{R F}$ linearly decreases from $1000 \mathrm{kV}$ to 700 $\mathrm{kV}$. The total time of the $2^{\text {nd }}$ rf pulse should be relatively short compared to the synchronous period. The starting time of the $2^{\text {nd }}$ pulse is varied for the optimization purpose, while the amplitude of the $2^{\text {nd }}$ pulse is kept at the constant value of $1000 \mathrm{kV}$, the rising time and falling time are kept the same, $10 \mu \mathrm{s}$, and the time at the flat top (1000 $\mathrm{kV}$ ) is also kept at the constant value of $80 \mu \mathrm{s}$. $\varepsilon_{L}$ with $95 \%$ of the beam and $\Delta p$ in rms $v s$. the starting time of the $2^{\text {nd }} \mathrm{rf}$ pulse, $T_{0}$, are shown as the black and blue curves in Fig. 2(a) respectively, while the $1^{\text {st }} \mathrm{rf}$ pulse is kept at its optimal setting. The charge transmission and BL vs. $T_{0}$ are shown as the black and blue curves in Fig. 2(b). It is clear that the optimal $T_{0}$ is $17.3 \mathrm{~ms}$. 
Finally, the optimal $V_{R F}$ curve, which covers the entire cycle, is applied in the ESME simulation. $V_{R F} v s$. time in a cycle is shown in Fig. 3(a). EPSILON $v s$. time is shown in Fig. 3(b). The phase and momentum projections at 8-GeV are shown in Figs. 3(c) and 3(d) respectively. $\Delta p$ in rms and $\varepsilon_{L}$ with $95 \%$ of the beam at $8-\mathrm{GeV}$ are 2.98 $\mathrm{MeV}$ and $0.061 \mathrm{eV} \cdot \mathrm{sec}$. Compared to the situation when the $V_{R F}$ curve in Fig. 1(a) is used, there is a $31 \%(\approx(0.088-0.061) / 0.088))$ reduction in $\varepsilon_{L}$ and a $17 \%$ reduction in $\Delta p$.

\section{Conclusions}

It requires Booster to be able to deliver $8-\mathrm{GeV}$ proton beams to Main Injector at the intensity of $4.5 \times 10^{12}$ per batch with $\varepsilon_{L}$ of $0.12 \mathrm{eV} \cdot \mathrm{sec}$ and $\Delta \mathrm{p}$ of $18 \mathrm{MeV}$ (with $95 \%$ of the beam) in order to achieve the antiproton production rate of $24 \times 10^{10}$ per hour. BR has been implemented to satisfy these requirements. However, BR frequently causes $\varepsilon_{L}$ growth whenever there are far more A stations than B stations, or vice versa, and the balancer doesn't work very well.[3] An alternate method via optimizing the $V_{R F}$ curve in an entire Booster cycle has been investigated using the ESME simulation. Especially, the rf manipulation at TR can bring a $31 \%$ reduction in $\varepsilon_{L}$ and a $17 \%$ reduction in $\Delta p$, so $\Delta p$ (in rms) of $2.98 \mathrm{MeV}$ and $\varepsilon_{L}$ (with $95 \%$ of the beam) of $0.061 \mathrm{eV} \cdot \mathrm{sec}$ at $8-\mathrm{GeV}$ can be achieved.

\section{References:}

[1] K. Koba, etc., "SLIP STACKING EXPERIMENTS AT FERMILAB MAIN INJECTOR”, FERMILAB-CONF-03-107.

[2] K. Koba and J. Steimel, "SLIP STACKING", FERMILAB-CONF-02-205.

[3] X. Yang, etc., "Reducing the Momentum Spread of the 8-GeV proton Beam via the Bunch Rotation in Booster", FERMILAB-FN-0769-AD, submitted.

[4] D. A. Edwards and M. J. Syphers, An Introduction to the Physics of High Energy Accelerators, 1993, John Wiley \& Sons, Inc.

[5] http://www-ap.fnal.gov/ESME/. 


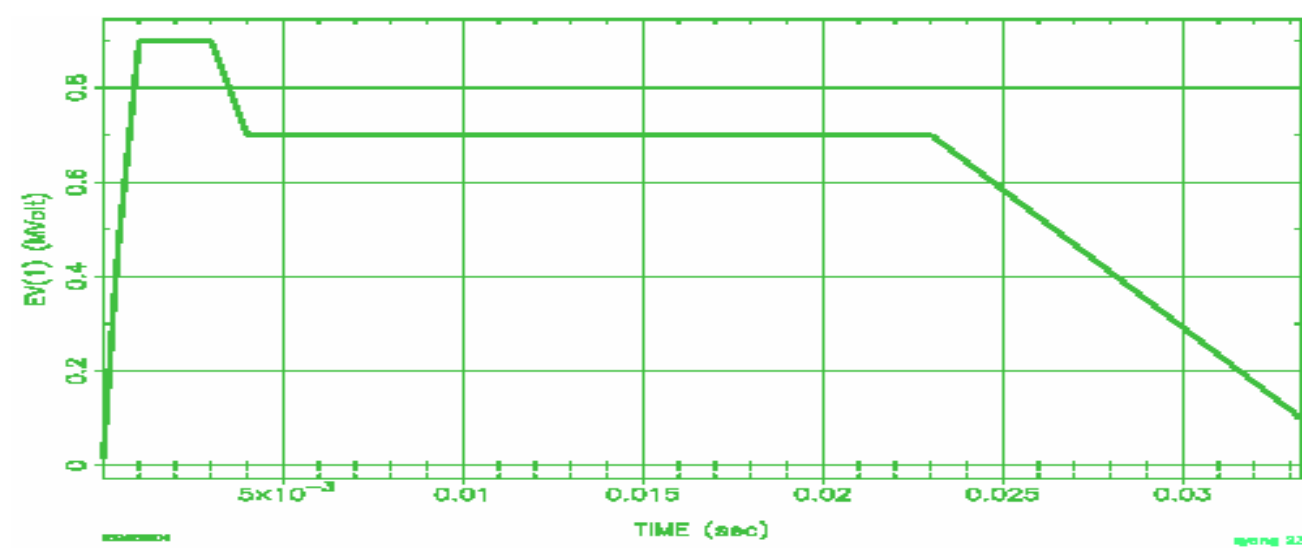

Fig. 1(a)

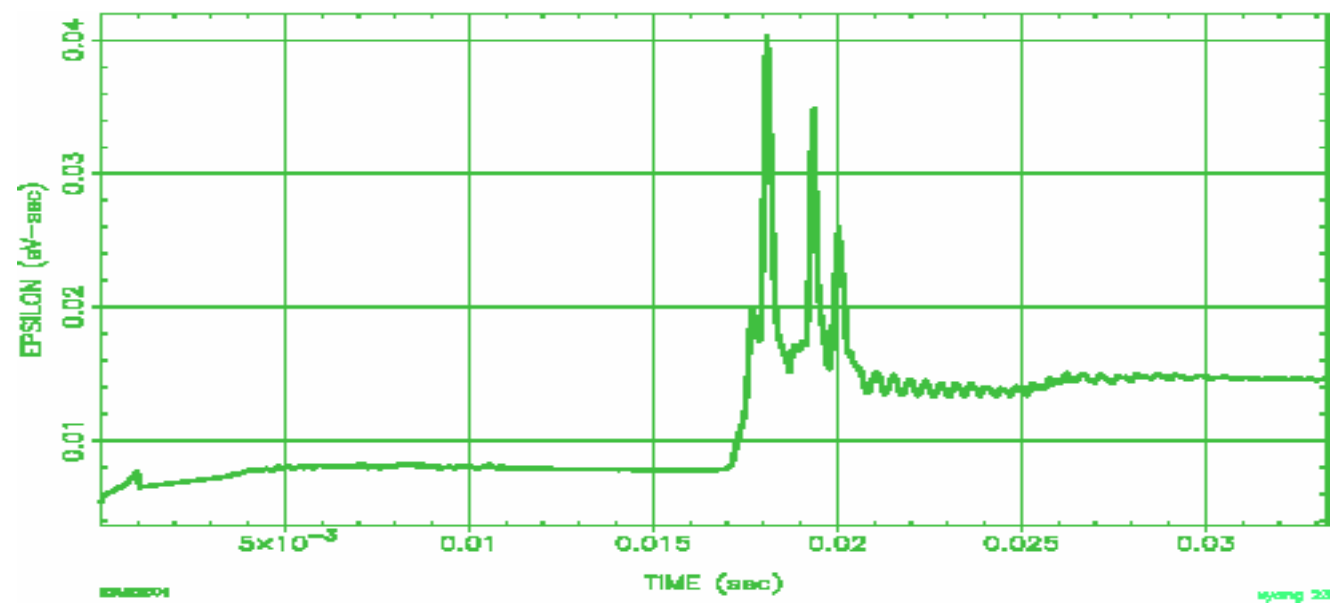

Fig. 1(b)

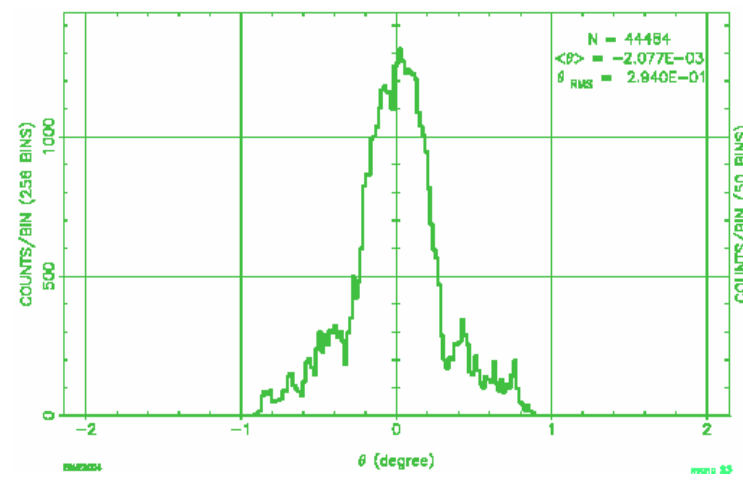

Fig. 1(c)

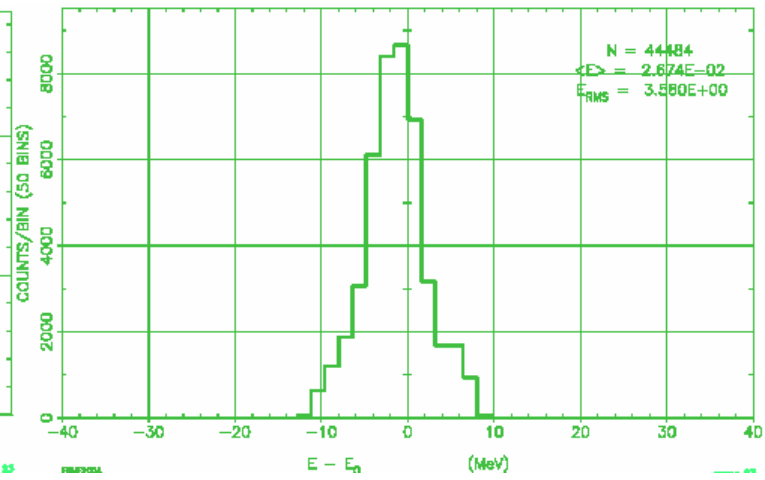

Fig. 1(d)

Fig. 1(a) $V_{R F} v s$. time in a cycle.

Fig. 1(b) EPSILON vs. time. For a Gaussian distribution, the area containing $95 \%$ of the beam, $\varepsilon_{L}$, is six time EPSILON.

Fig. 1(c) the phase projection at $8-\mathrm{GeV}$.

Fig. 1(d) the momentum projection at $8-\mathrm{GeV}$. 


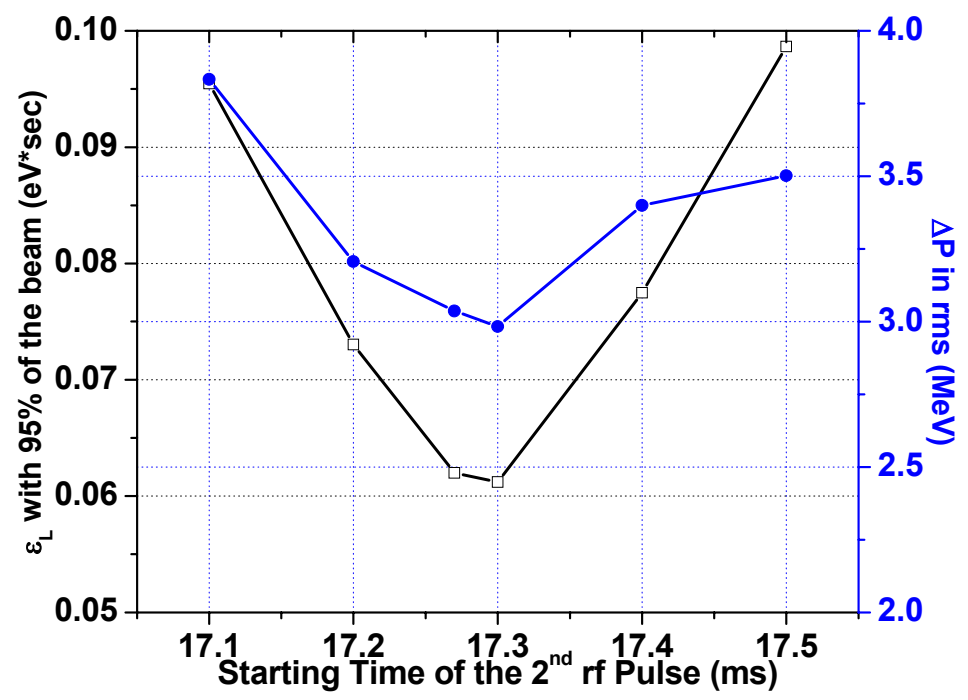

Fig. 2(a)

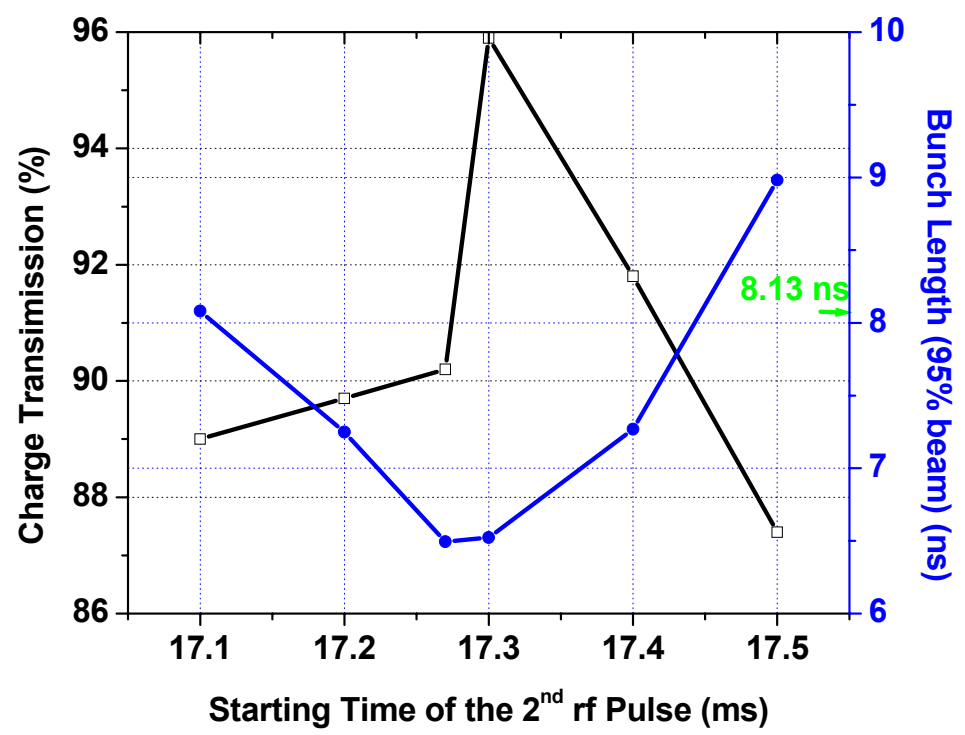

Fig. 2(b)

Fig. 2(a) $\varepsilon_{L}$ with $95 \%$ of the beam and $\Delta p$ in rms $v s$. the starting time of the $2^{\text {nd }}$ rf pulse, $T_{0}$, are shown as the black and blue curves respectively.

Fig. 2(b) the charge transmission and BL vs. $T_{0}$ are shown as the black and blue curves. 


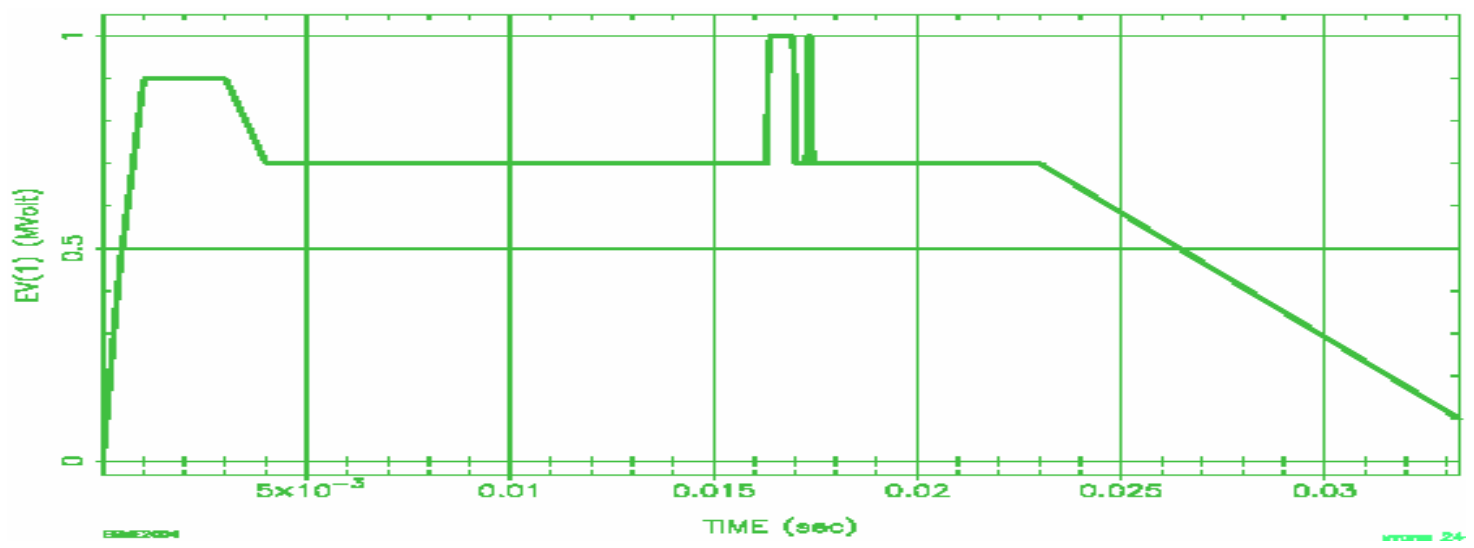

Fig. 3(a)

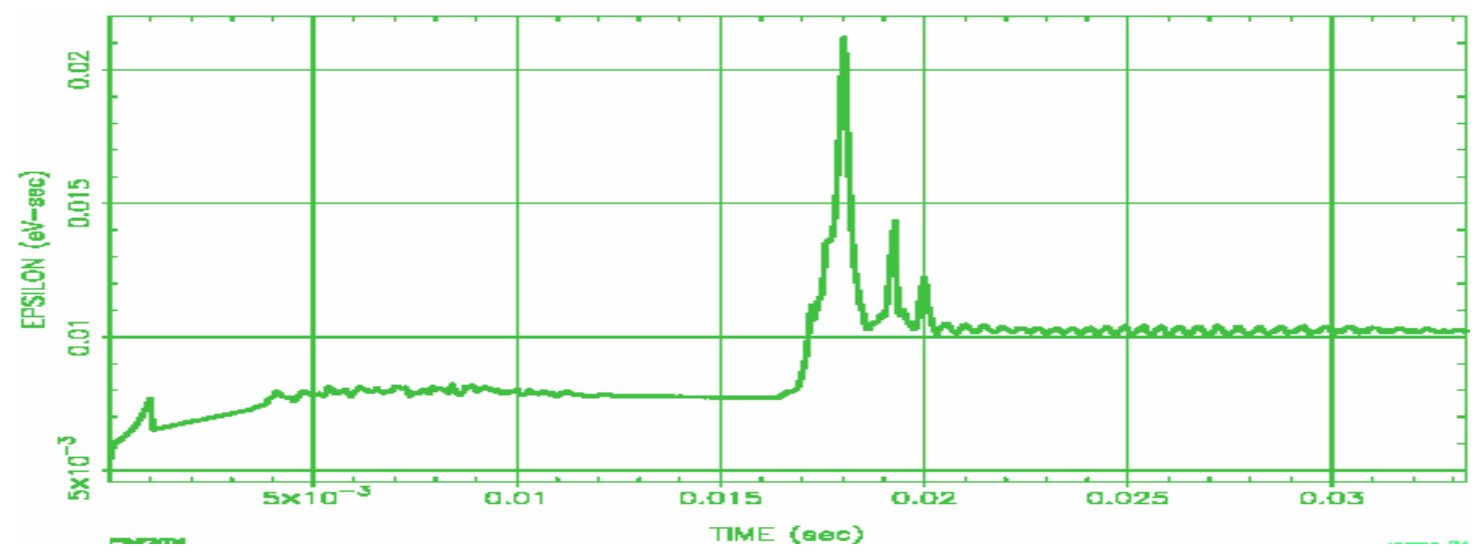

Fig. 3(b)

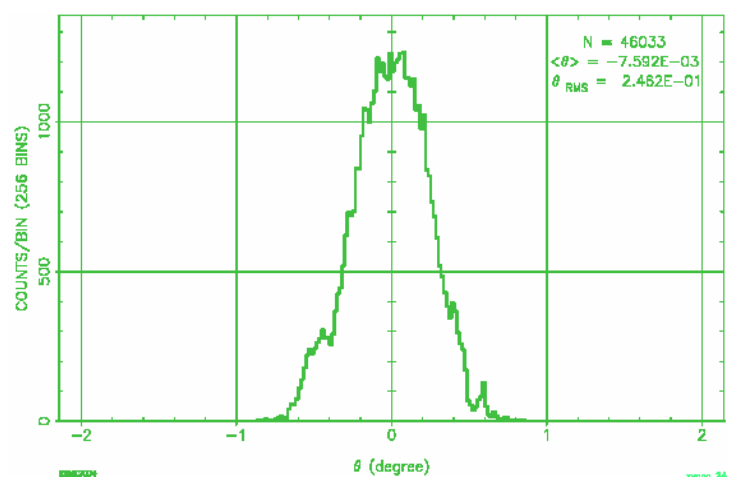

Fig. 3(c)

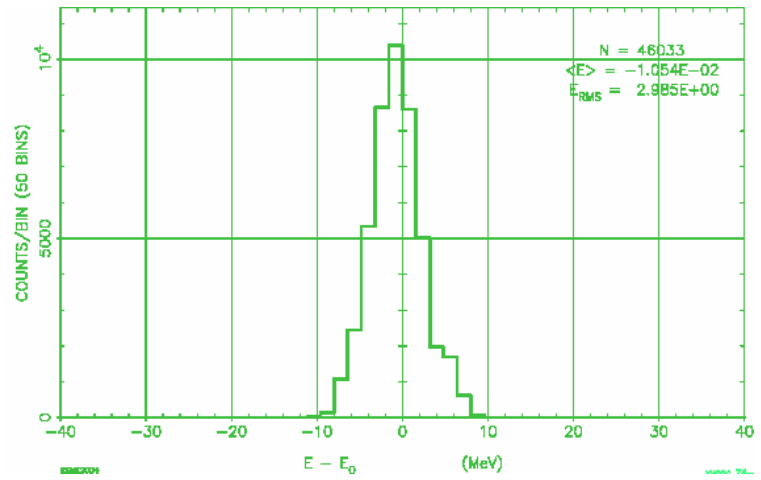

Fig. 3(d)

Fig. 3(a) $V_{R F} v s$. time in a cycle.

Fig. 3(b) EPSILON vs. time. For a Gaussian distribution, the area containing $95 \%$ of the beam, $\varepsilon_{L}$, is six time EPSILON.

Fig. 3(c) the phase projection at $8-\mathrm{GeV}$.

Fig. 3(d) the momentum projection at $8-\mathrm{GeV}$. 